\title{
Lungenrundherde immer bronchoskopisch abklären?
}

Die Bedeutung der präoperativen Bronchoskopie in der Diagnostik solitärer Lungenrundherde ist umstritten. Die Pneumologen fordern sie, da mit dieser Methode besser als mit der Computertomografie Tumore in den Bronchien entdeckt werden können. In Ermangelung einer Bronchoskopie sind die Onkologen häufig schon mit der radiologischen Bildgebung zufrieden, und die Thoraxchirurgen empfinden die Bronchoskopie $u$. U. als lästige Zeitverzögerung.

— Die Autoren der vorliegenden Arbeit untersuchten an ihrem Krankengut, wie häufig die präoperative Bronchoskopie bei Patienten mit solitären Lungenrundherden gegenüber anderen Methoden einen zusätzlichen Erkenntnisgewinn erbrachte.

Zwischen 2004 und 2007 wurden prospektiv 225 Patienten mit einem solitären Rundherd auch bronchoskopisch untersucht.

Von den rekrutierten Patienten litten 80,5\% an einem Lungenkarzinom, 7,6\% hatten eine intrapulmonale Metastase eines extrapulmonalen Karzinoms. 12\% aller Herde waren benigne.

Die Bronchoskopie erbrachte bei $4,4 \%$ der Patienten eine mit anderen Methoden nicht detektierbare Veränderung in den Bronchien und in $41 \%$ aller Fälle eine ätiologische Klärung. Bei 46,5\% aller Herde konnten die Tumoren histologisch oder zytologisch mittels fle-

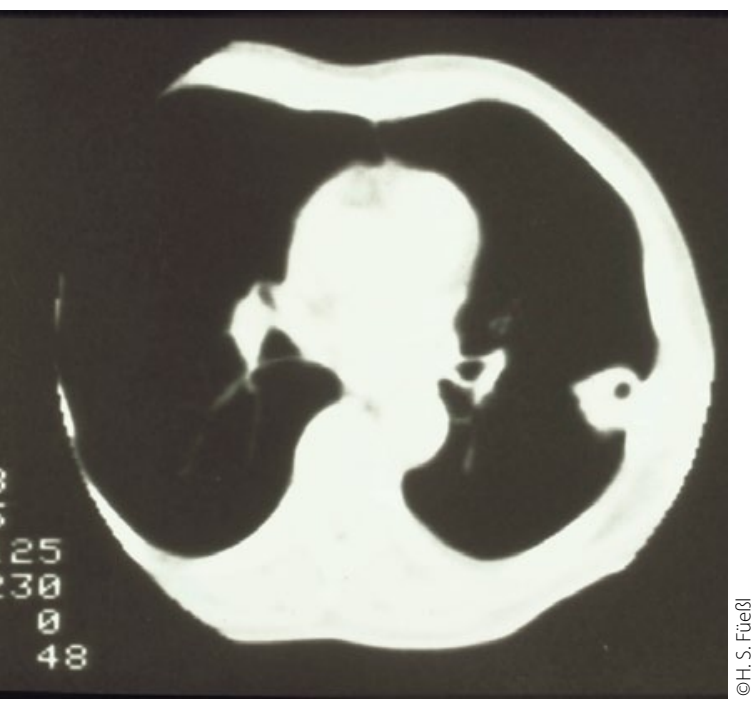

Wandnahes Bronchialkarzinom in der Computertomografie. xibler Bronchoskopie gesichert werden. In fünf Fällen musste wegen des bronchoskopischen Ergebnisses das initial avisierte chirurgische Vorgehen geändert werden.

\section{- C. Schwarz et al.}

Value of flexible bronchoscopy in the pre-operative work-up of solitary pulmonary nodules. Eur. Respir. J. 41:177-182, 2013

\section{Kommentar}

Die Autoren schlussfolgern, dass die fünf von 225 Fällen, in denen das präoperative bronchoskopische Ergebnis die chirurgische Strategie maßgeblich veränderte, eine reguläre Bronchoskopie im Rahmen des Stagings immer rechtfertigen. So konnte in zwei Fällen eine bakterielle Pneumonie als Ursache der intrapulmonalen Verschattung diagnostiziert werden, die lediglich eine Antibiotikagabe und keine Resektionschirurgie erforderlich machte. Es bestehe somit kein Zweifel, dass zur präoperativen Diagnostik pulmonaler Rundherde die Bronchoskopie einschließlich der heute zur Verfügung stehenden ultraschallgestützten endobronchialen Punktionsdiagnostik (EBUS) als conditio sine qua non des Stagings dazugehört.

Einige wichtige Aspekte muss man bei dieser Studie allerdings beachten. Erstens standen den Autoren zum o. g. Untersuchungszeitpunkt keine PET-CT- und EBUS-Untersuchungsmöglichkeit zur Verfügung, die das bronchoskopische Ergebnis möglicherweise noch zusätzlich hätten positiv beeinflussen können. Zweitens muss man der insgesamt und auch im Vergleich zu früheren Publikationen hohen bronchoskopischen Trefferquote zugutehalten, dass die Untersuchung an einer pneumologischen Fachklinik mit jahrzehntelanger Bronchoskopie-Erfahrung durchgeführt wurde.

A. GILLISSEN -

\section{ACHTUNG:}

\section{Hier muss der Dummy durch eine Anzeige} ersetzt werden !! 